\title{
Absence of False Acetabulum is a Potent Indicator for Predicting the Use of Subtrochanteric Osteotomy in Total Hip Arthroplasty for High Dislocated Hips
}

Jing-yang Sun

Chinese PLA General Hospital

Guo-qiang Zhang

Chinese PLA General Hospital

Yin-qiao Du

Chinese PLA General Hospital

Jun-min Shen

Chinese PLA General Hospital

Yong gang Zhou ( $\nabla$ ygzhou301@163.com)

Chinese PLA General Hospital https://orcid.org/0000-0002-7304-4691

\section{Yan Wang}

Chinese PLA General Hospital

\section{Research article}

Keywords: high dislocated hips, false acetabulum, subtrochanteric osteotomy, developmental dysplasia of the hip, total hip arthroplasty

Posted Date: May 12th, 2020

DOI: https://doi.org/10.21203/rs.3.rs-26221/v1

License: (c) (i) This work is licensed under a Creative Commons Attribution 4.0 International License. Read Full License 


\section{Abstract}

Introduction: This study aimed to evaluate the predictive values of false acetabulum for assessment of whether to use subtrochanteric osteotomy.

Materials and Methods: We retrospectively included a total of 182 patients ( 232 hips) affected by Crowe type IV developmental dysplasia who underwent primary THA with modular cementless stem from April 2008 to May 2019 in our institution. Based on radiographs and operative notes, we found 175 hips were performed with subtrochanteric osteotomy and 57 without subtrochanteric osteotomy, which was named (subtrochanteric osteotomy) STO group and non-STO group, respectively. The predictive values of absence of false acetabulum and distalization of greater trochanter were analyzed using receiver operating characteristic (ROC) curves.

Results: ROC curves showed that absence of false acetabulum and distalization of greater trochanter had the AUCs of 0.957 and 0.987 , respectively. And there was no statistical significance in the difference of these two AUCs $(P=0.392)$. The optimal threshold for the distalization of greater trochanter was $4.83 \mathrm{~cm}$, which resulted in a sensitivity, specificity, positive predictive value (PPV), and negative predictive value (NPV) of $92.6 \%, 100 \%, 100 \%$, and $81.4 \%$, respectively. For the absence of false acetabulum, the sensitivity, specificity, PPV, and NPV were $94.9 \%, 96.5 \%, 98.8 \%$, and $85.9 \%$, respectively.

Conclusions: The absence of false acetabulum may be a potent indicator in predicting the use of subtrochanteric osteotomy in high dislocated hips.

\section{Introduction}

Total hip arthroplasty (THA) in developmental dysplasia of the hip (DDH) is technically very demanding procedure due to morphological variations $[1,2]$. In the setting of severe dysplasia (Crowe type IV or Hartofilakidis type C) [3,4], high riding hip centers and soft tissue contractures are quite likely to be encountered by surgeons [5]. Therefore, femoral shortening may be necessary for anatomic placement of the acetabular component and to avoid neurovascular complications. Several methods have been described for femoral shortening osteotomy in Crowe type IV hips, including greater trochanteric osteotomy, lesser trochanteric osteotomy, subtrochanteric osteotomy, and supracondylar osteotomy [6-9].

Greater trochanteric osteotomy can present favorable exposure of the acetabulum and proximal femur and restore a substantial abductor lever arm with distal advancement. Meanwhile, it also brings the concern of difficult reattachment and nonunion of the greater trochanter, which could adversely affect abductor strength and hip function $[6,10]$. Lesser trochanteric osteotomy facilitates femoral shortening by sequential resection of femoral neck. However, it sacrifices the best proximal bony architecture and is associated with abductor weakness [7]. The supracondylar osteotomy may increases the wound surface and is more often indicated when there is a concomitant severe valgus deformity of the knee [9]. Subtrochanteric osteotomy can be performed using a variety of techniques including transverse, oblique, Z-shaped, or double chevron osteotomy [11-14]. Muratli et al. demonstrated that there was no difference 
in the stability of these four techniques during load testing in a biomechanical lab setting [15]. And compared to other osteotomies, subtrochanteric transverse shortening osteotomy may be recommended due to technical simplicity, minimal periosteal damage at the osteotomy site, preservation of the proximal femoral metaphysis, and relatively short learning curve for precise performance.

Over the years, several methods emerged aiming for avoidance of femoral shortening, including intraoperative injection of muscle relaxant, more extensive soft tissue release, leverage and progressive femoral lowering by iliofemoral distraction [16-19]. And as demonstrated in some studies, THA without subtrochanteric shortening osteotomy is possible in Crowe type IV hips, which can also provide good clinical outcomes and acceptable complication rates [20,21]. In some studies, subtrochanteric osteotomy was recommended when the lower limb would be lengthened more than $3 \mathrm{~cm}, 4 \mathrm{~cm}$, or $5 \mathrm{~cm}$ in dislocated hips to decrease the risk of excessively stretching sciatic nerve [22-24]. However, there is no consensus on the maximum safe amount of limb lengthening. Besides, multimodal intraoperative monitoring has been described to detect early warning signs of nerve injury and to guide the use of subtrochanteric osteotomy [25]. Interestingly, the radiographic measurements of both Xu et al. [26] and Ma et al. [27] confirmed that proximal femur in high dislocated hips absent of false acetabulum was narrower, with little metaphysis flare to mate with the stem, and often migrated more superiorly than that in hips with false acetabulum formation. And they presumed that limb of the former type might gain more lengthening when repositioning the hip into the anatomical center of rotation, thus making subtrochanteric osteotomy more likely to be performed.

Considering the potential relation between false acetabulum and subtrochanteric osteotomy, we hypothesized that absence of false acetabulum might be a useful indicator for predicting the use of subtrochanteric osteotomy in total hip arthroplasty for Crowe type IV DDH. The aim of this study was to evaluate the predictive values of false acetabulum for assessment of whether to use subtrochanteric osteotomy.

\section{Methods}

\section{Study population}

After the Institutional Review Board approval, we retrospectively reviewed 242 consecutive patients (305 hips) affected by Crowe type IV DDH who underwent primary THA with modular cementless stem (SROM, DePuy, Warsaw, Indiana) from April 2008 to May 2019 in our institution. Exclusion criteria were (1) previous femoral osteotomy, or (2) previous hip pyogenic arthritis, or (3) proximal placement of the cup component, or (4) use of conical sleeve, or (5) inadequate postoperative anteroposterior (AP) radiographs. Finally, a total of 182 patients (232 hips) were included in the study (Fig. 1).Demographics and clinical information including age, gender, weight, height, and operative note were collected from our electronic medical records. 


\section{Radiographic assessment}

Standardized digital, calibrated AP hip and full-length standing AP radiographs were acquired both preoperatively and postoperatively [28].The performance of subtrochanteric osteotomy was validated by osteotomy line on images of the second day after surgery and also by reference to operative notes (Fig.2). Radiographic assessment contained the recognition of false acetabulum and the measurement of distalization of greater trochanter. The presence of false acetabulum was defined as a fossa that obstructs the femur from higher dislocation and does not overlap with the true acetabulum (Fig.3). The distalization of greater trochanter was calculated by vertical height difference between the pre-and postoperative tip of the greater trochanter, which was perpendicular to the inter-teardrop line. Before initiation of the study, all observers reached an agreement on criteria for radiographic assessment. Removing all identifying marks, each part of radiographic assessment was accomplished by two observers independently in random order, who were not involved in index surgery. Then at least one month later, observers repeated their readings without knowledge of the previous results. All measurements were conducted by using Digimizer v5.4 (Acacialaan, Belgium). Inter-observer variability was measured by comparing the mean value of two observers on each occasion, while intra-observer reliability was determined by comparing the two reviews of each observer.

\section{Surgical procedure}

All operations were performed by one senior surgeon under general anesthesia in the lateral decubitus position. The posterolateral approach was used in each case. Joint capsulectomy, gluteal sling release, and iliopsoastenotomy was performed. In order to use ceramic on ceramic bearing, the cup (range 44-46 $\mathrm{mm}$ ) was implanted at the anatomic position by reaming the acetabulum posteriorly and inferiorly [29]. Two or three screws were used to augment primary stability of the cup. No additional stabilization such as femoral head autograft, cage or ring was used to support the acetabulum. After the cup implantation, the femur was internally rotated $90^{\circ}$ and the femoral canal was prepared using the dedicated reamer for the S-ROM stem. First, we reamed the femoral canal until the maximum cortical contact was reached distally. Then conical and triangular reaming of the metaphysis was performed to prepare for the proximal sleeve. With trial seated in the femur, we measured the final vertical distance from femoral head to cup under constant and vigorous traction. If hip reduction with a femoral trial stem was impossible, a subtrochanteric osteotomy would be performed for femoral shortening. The osteotomy position was planned to be adjacent enough to the end of the proximal sleeve, approximately corresponding to $1-2 \mathrm{~cm}$ beneath the lesser trochanter, which was able to provide sufficient engagement of the implant to stabilize the osteotomy site. Prophylactic cerclage wires were placed both proximally and distally around the fragment of the planned osteotomy. Also, a longitudinal line along the femoral diaphysis was marked by electrocautery prior to osteotomy to determine the rotation. After removing the trial, a transverse osteotomy was performed, by resection of a length of the femur below the lesser trochanter. The length of the removed bone stock was based on the distance we measured before, leaving a scope of $1-1.5 \mathrm{~cm}$ with surgeon's discretion. After completion of osteotomy, a final preparation of the femur including repeated 
reaming and broaching was undertaken, until optimal cortical contact was achieved especially distal to the osteotomy site. Then trial reduction was performed again. If impossible, additional bone was incrementally resected at the osteotomy site until reduction was achieved. After trial reduction, stability, limb length and soft tissue tension were evaluated. Intraoperative measurement of limb length discrepancy (LLD) was performed by palpating the inferior point of bilateral patella. Mild LLD could be adjusted by means of the modifications of head/neck length and stem depth in femur. Finally, the definitive femoral component was implanted with the rotational alignment of femoral stem adjusted to allow approximately $30-50^{\circ}$ of combined anteversion. During the stem insertion, compression of the osteotomy site was obtained without any gaps and no rotational adjustment of the bone segment was performed. At the end of the surgery, motion in abduction was assessed to evaluate the necessity of a percutaneous partial adductor tenotomy. Postoperatively, patient's hip and knee were maintained in flexion for several days to relax the sciatic nerve and reduce tension of soft tissue. In cases of bilateral Crowe type IV dysplasia, apart from consideration for safe reduction, limb length would be equalized through the amount of bone resection and the modularity of implant.

Patients were followed up in regular intervals at 3 months, 6 months, and yearly after surgery. Clinical information was collected including LLD measured on full-length standing AP radiograph (from the base of teardrop to the center of plafond), and occurrence of nerve injury and dislocation.

\section{Statistical Analysis}

All statistical analyses were performed using SPSS version 26.0 (IBM Inc., Armonk, New York) and Microsoft Excel. Assessment of inter- and intra-observer consistency was accomplished by the use of the intraclass correlation coefficient (ICC). Agreement was graded as slight (ICC $=0$ to 0.2 ), fair (ICC $=0.21$ to 0.40 ), moderate (ICC $=0.41$ to 0.60 ), substantial (ICC $=0.61$ to 0.80 ) or almost perfect (ICC $=0.81$ to 1.0 ). Categorical variables were presented as frequencies and continuous variables as means with ranges or median with interquartile range (IQR). Continuous variables were assessed using t-test or Mann-Whitney test, whereas categorical variables were analyzed using Chi-square test or Fisher's exact test. Receiver operating characteristic (ROC) curves were generated to determine the predicting value of each indicator for the assessment of subtrochanteric osteotomy. The area under the curve (AUC) and 95\% confidence interval $(\mathrm{Cl})$ were calculated. The discriminatory value of curves was interpreted as excellent (0.9 to 1$)$, good ( 0.8 to 0.89 ), fair ( 0.7 to 0.79 ), poor ( 0.6 to 0.69$)$, or as failing or having no discriminatory capacity ( 0.5 to 0.59$)$. The comparison of AUCs was analyzed by $Z$ test. The optimal threshold for indicator as a predicting tool for subtrochanteric osteotomy was determined using the Youden index. The sensitivity, specificity, positive predictive value (PPV), and negative predictive value (NPV) of the indicators were calculated. $P$ value of $<0.05$ was considered significant.

\section{Results}


On the basis of radiographs and operative notes, 175 hips with subtrochanteric osteotomy and 57 hips without subtrochanteric osteotomy were included in the study, which was named STO group and nonSTO group for short, respectively. Demographic data are summarized in Table 1.

Table 1

Demographic data of two groups

\begin{tabular}{|llll|}
\hline & STO group $(\mathrm{n}=175)$ & Non-STO group $(\mathrm{n}=57)$ & P-value \\
\hline Male/female sex, $\mathrm{n}$ & $18 / 157$ & $2 / 55$ & 0.172 \\
\hline Median age, years(IQR) & $41(29$ to 50$)$ & $40(29$ to 48$)$ & 0.521 \\
\hline Median height, $\mathrm{m}(\mathrm{IQR})$ & $1.58(1.53$ to 1.60$)$ & $1.55(1.50$ to 1.60$)$ & 0.371 \\
\hline Median weight, $\mathrm{kg}(\mathrm{IQR})$ & $56.5(51.0$ to 63.5$)$ & $53.0(50.7$ to 60.0$)$ & 0.205 \\
\hline Median body mass index, $\mathrm{kg} / \mathrm{m}^{2}(\mathrm{IQR})$ & $22.5(21.1$ to 24.1$)$ & $22.5(21.1$ to 23.4$)$ & 0.446 \\
\hline Unilateral/bilateral, $\mathrm{n}$ & $82 / 93$ & $48 / 9$ & $<0.001$ \\
\hline Median follow-up, months(IQR) & $8(6$ to 24$)$ & $8(6$ to 34$)$ & 0.182 \\
\hline
\end{tabular}

In the STO and non-STO groups, the proportion of false acetabulum formation was $9 / 175$ and 55/57 $(P<0.001)$, respectively. Inter-observer variability analysis revealed ICC of $0.977(95 \% \mathrm{Cl}, 0.676$ to 0.993$)$ (almost perfect) for the measurement of distalization of greater trochanter. Intra-observer reliability analysis revealed ICC of $0.981(95 \% \mathrm{Cl}, 0.862$ to 0.993$)$ (almost perfect) for observer $\mathrm{A}$, and $0.975(95 \% \mathrm{Cl}$, 0.800 to 0.991 ) (almost perfect) for observer B. Distalization of greater trochanter was $6.86 \mathrm{~cm}$ (3.46 to $11.39)$ in the STO group and $3.17 \mathrm{~cm}(1.13$ to 4.80$)(P<0.001)$ in the non-STO group. It was also evaluated in hips with or without false acetabulum, with the results of $3.41 \mathrm{~cm}(1.13$ to 8.05$)$ and $6.93 \mathrm{~cm}$ (3.18 to 11.39) $(P<0.001)$.

The ROC curves showed that distalization of greater trochanter and absence of false acetabulum had the AUCs of 0.987 and 0.957 , respectively, indicating that they had excellent predictive value (Fig. 4). And there was no statistical significance in the difference of these two AUCs $(P=0.392)$.

Based on our data, the optimal threshold for the distalization of greater trochanter was $4.83 \mathrm{~cm}$, which resulted in a sensitivity, specificity, PPV, and NPV of $92.6 \%, 100 \%, 100 \%$, and $81.4 \%$, respectively. For the absence of false acetabulum, the sensitivity, specificity, PPV, and NPV were $94.9 \%, 96.5 \%, 98.8 \%$, and $85.9 \%$, respectively.

At the last follow-up, seven dislocations (at 1 months, 2 months, and 3 months) occurred in 7 patients (4 in STO group and 3 in non-STO group). Five hips were treated by closed reduction and 2 by open reduction). Seven patients (6 in STO group and 1 in non-STO group) developed femoral nerve palsy with skin numbness on the frontal thigh or tibia and all recovered in a year. Eighty-six postoperative full-length standing AP radiographs were acquired. LLD was $<1 \mathrm{~cm}$ in $68 / 86,1$ to $2 \mathrm{~cm}$ in $17 / 86$, and $>2 \mathrm{~cm}$ in $1 / 86$. 


\section{Discussion}

Due to morphological variations of high dislocated hips, it is generally difficult for reduction especially when the cup is reconstructed at the anatomical position [5]. Current studies mainly focused on the outcomes of THA with or without femoral shortening [16, 18, 20,21,30,31]. There is no consensus as to when it is requisite to perform femoral shortening. In this retrospective study, we found that absence of false acetabulum was a potent indicator for predicting the use of subtrochanteric osteotomy, with excellent sensitivity and specificity.

Previous studies have recommended femoral shortening to prevent excessive limb lengthening and subsequent potential nerve injury. And the proposed threshold of limb lengthening varied greatly among different authors, mainly with the range from 3 to $5 \mathrm{~cm}$ [22-24]. The estimation of expected limb lengthening was accomplished by preoperative templating on plain radiographs in most literatures $[31,32]$. However, it has been demonstrated that the femur in dysplastic hips had a more anteverted canal than ones with primary osteoarthritis [33]. Therefore, a two-dimensional imaging may set up pitfalls of defining implant size and insertion depth in high dislocated hips. In this study, we calculated vertical height difference between the pre-and postoperative tip of the greater trochanter, which actually indicated the migration of proximal femur during reduction of the hip. As an indicator, distalization of greater trochanter performed very well in predicting the use of subtrochanteric osteotomy and had an excellent AUC (0.987). The optimal threshold was calculated to be $4.83 \mathrm{~cm}$, which was close to the value $(5 \mathrm{~cm})$ proposed by Higuchi et al. [23] Though more accurate than X-raytemplating, the distalization of greater trochanter is of no avail to clinical practice due to its unavailability before surgery.

Interestingly, another indicator, the absence of false acetabulum, also performed well and had a similar predictive accuracy with limb lengthening. As was described in Hartofilakidis[4] and Eftekhar[34] classification systems, high dislocated hips were subdivided into two different types depending on the presence of false acetabulum. With the aid of those subtypes, they expected to predict the acetabular bone deficiencies encountered during THA and plan for reconstruction method. Afterwards, Xu et al. [26] and $\mathrm{Ma}$ et al. [27]measured the canal flare index and dislocation height of femur in high dislocated hips on anteroposterior radiographs. Both of their results showed that in hips without false acetabulum, the proximal femur was more like a stove-pipe, and with more amount of dislocation height. It was speculated that the morphological difference between the two subtypes could be attributed to the stress stimulus provided by false acetabulum according to Wolf's law. Because of the anatomical abnormalities, the center of femoral head is likely to be restored to a higher position with a suspended stem. Along with the condition of higher dislocation, femoral shortening, represented by subtrochanteric osteotomy, is generally necessary to be performed to equalize limb lengths and avoid excessive lengthening [35]. Based on our data, the PPV of the absence of false acetabulum was $98.8 \%$, indicating an extremely high percentage of the use of subtrochanteric osteotomy. In contrast, 55/64 hips with false acetabulum achieved reduction without femoral shortening. Possible explanation for this substantial NPV (85.9\%) may be lower dislocation height and more insertion depth of stem in those hips, which give less 
contribution to the limb lengthening. More studies like computerized three-dimensional planning may be needed to recognize the effect of false acetabulum on femoral morphology.

Our results of distalization of greater trochanter may indirectly identify the role of false acetabulum for predicting femoral shortening. The mean distalization was $3.41 \mathrm{~cm}$ (1.13 to 8.05) in hips with false acetabulum and $6.93 \mathrm{~cm}$ (3.18 to 11.39) in hips without false acetabulum. Same tendency was observed in the studies by Nagoya et al. [36] and Fujishiro et al. [37]. Nagoya et al. included 20 hips with Crowe type IV dislocation of which 8 were previously treated with femoral osteotomy. Their results showed the mean distalization of the greater trochanter was $3.63 \mathrm{~cm}$ in patients with iliofemoral osteoarthritis (OA), and $6.78 \mathrm{~cm}$ in patients without iliofemoral $\mathrm{OA}$, which were close to our data. Fujishiro et al. conducted a similar study with a larger scale (70 hips) and used S-ROM femoral prosthesis uniformly. In their study, the mean distalization of the greater trochanter of hips with or without iliofemoral OA were $4.93 \mathrm{~cm}$ and $7.15 \mathrm{~cm}$. It is important to note that iliofemoral OA described in their essays does not equate to the presence of false acetabulum. According to our definition, it is not the pseudarthrosis between the pelvic wall and the femoral head and/or lesser trochanter, but a fossa providing load stimulus and obstructing higher dislocation that counts. Perhaps the heterogeneity of their subjects may partially explain why the migration of proximal femur in hips with iliofemoral OA is a little greater than our data.

The demographic data revealed that hips in bilateral cases occupied a bigger proportion in STO-group than in non-STO group. Further statistical analysis showed that 88/102 hips in bilateral cases were absent of false acetabulum, significantly more than that in unilateral cases $(80 / 130)(p<0.001)$. So the difference on unilateral and bilateral sides could be explained by the presence of false acetabulum.

There are several limitations in this study. First, this was a retrospective study. Fortunately, we mainly relied on the radiographs with less effect from recall bias. Second, several factors such as history of femoral osteotomy or pyogenic arthritis, and use of conical stem were excluded, aiming to determine the effect of false acetabulum by itself. However, these factors are thought to influence the hip reduction. Therefore, a comprehensive, multivariate analysis may be needed. Additionally, this study was designed as a diagnostic test. We did not present much information about the outcome of THA with or without subtrochanteric osteotomy. We are now planning to carry out another study on the limb equalization after this procedure. Finally, it was a single-institution, single-surgeon study with use of a single femoral prosthesis, and, as a result, has limited external validity. However, with the excellent predictive accuracy, subtypes based on the presence of false acetabulum can indeed remind arthroplasty surgeons of potential different interventions on femoral side in Crowe type IV hips.

\section{Conclusions}

The absence of false acetabulum may be a promising and potent indicator in predicting the use of subtrochanteric osteotomy in high dislocated hips. However, there are some other factors influencing hip reduction. A multivariate analysis is required to further validate predictive values of false acetabulum. 


\section{Declarations}

\section{Acknowledgements}

The authors would like to thank all staff from the participating departments and clinics.

\section{Funding}

This research did not receive any specific grant from funding agencies in the public, commercial, or notfor-profit sectors.

\section{Compliance with Ethical Standards}

\section{Conflict of interest}

The authors declare that they have no conflict of interest.

\section{Ethical approval}

This retrospective review study involving human participants was in accordance with the ethical standards of the institutional and national research committee and with the 1964 Helsinki Declaration and its later amendments or comparable ethical standards. The Human Investigation Committee (IRB) of our hospital approved this study.

\section{Informed consent}

Informed consent was obtained from all individualparticipants included in the study.

\section{Author's contribution}

Yong-gang Zhou and Yan Wang contributed to the study conception and design. Material preparation, data collection and analysis were performed by Yin-qiao Du, and Jun-min Shen. The first draft of the manuscript was written by Jing-yang Sun and Guo-qiang Zhang. All authors commented on previous versions of the manuscript. All authors read and approved the final manuscript.

\section{References}


1. Rogers BA, Garbedian S, Kuchinad RA, Backstein D, Safir O, Gross AE (2012) Total hip arthroplasty for adult hip dysplasia. J Bone Joint Surg [Am]94: 1809-21. http://doi.org/10.2106/JBJS.K.00779

2. Kosuge D, Yamada N, Azegami S, Achan P, Ramachandran M (2013) Management of developmental dysplasia of the hip in young adults: current concepts. Bone Joint J95-B: 732-7. http://doi.org/10.1302/0301-620X.95B6.31286.

3. Crowe JF, Mani VJ, Ranawat CS (1979) Total hip replacement in congenital dislocation and dysplasia of the hip. J Bone Joint Surg [Am] 61: 15-23.

4. Hartofilakidis G, Yiannakopoulos CK, Babis GC (2008) The morphologic variations of low and high hip dislocation. Clin Orthop Relat Res 466: 820-4. http://doi.org/10.1007/s11999-008-0131-9.

5. Bicanic G, Barbaric K, Bohacek I, Aljinovic A, Delimar D (2014) Current concept in dysplastic hip arthroplasty: Techniques for acetabular and femoral reconstruction. World J Orthop 5: 412-24. http://doi.org/10.5312/wjo.v5.i4.412.

6. Charnley J. The long-term results of low-friction arthroplasty of the hip performed as a primary intervention (1972)J Bone Joint Surg [Br] 54: 61-76.

7. Bao N, Meng J, Zhou L, Guo T, Zeng X, Zhao J (2013) Lesser trochanteric osteotomy in total hip arthroplasty for treating CROWE type IV developmental dysplasia of hip. Int Orthop 37: 385-90. http://doi.org/10.1007/s00264-012-1758-4.

8. Li C, Zhang C, Zhang M, Ding Y (2014) Comparison of transverse and modified subtrochanteric femoral shortening osteotomy in total hip arthroplasty for developmental dysplasia of hip: a metaanalysis. BMC Musculoskelet Disord 15: 331. http://doi.org/10.1186/1471-2474-15-331.

9. Koulouvaris P, Stafylas K, Sculco T, Xenakis T (2008) Distal femoral shortening in total hip arthroplasty for complex primary hip reconstruction. A new surgical technique. J Arthroplasty 23 : 992-8. http://doi.org/10.1016/j.arth.2007.09.013.

10. Hartofilakidis G, Babis GC, Georgiades G, Kourlaba G (2011) Trochanteric osteotomy in total hip replacement for congenital hip disease. J Bone Joint Surg [Br] 93: 601-7. http://doi.org/10.1302/0301-620X.93B5.24780

11. Masonis JL, Patel JV, Miu A,Bourne RB, McCalden R, Macdonald SJ, Rorabeck CH (2003) Subtrochanteric shortening and derotational osteotomy in primary total hip arthroplasty for patients with severe hip dysplasia: 5-year follow-up. J Arthroplasty 18: 68-73. http://doi.org/10.1054/arth.2003.50104.

12. Dallari D, Pignatti G, Stagni C, Giavaresi G, Del Piccolo N, Rani N, Veronesi F, Fini M (2011) Total hip arthroplasty with shortening osteotomy in congenital major hip dislocation sequelae. Orthopedics 34: e328-33. http://doi.org/10.3928/01477447-20110627-14.

13. Sener N, Tözün IR, Aşik M (2002) Femoral shortening and cementless arthroplasty in high congenital dislocation of the hip. J Arthroplasty 17: 41-8. http://doi.org/10.1054/arth.2002.27672.

14. Becker DA, Gustilo RB (1995) Double-chevron subtrochanteric shortening derotational femoral osteotomy combined with total hip arthroplasty for the treatment of complete congenital dislocation 
of the hip in the adult. Preliminary report and description of a new surgical technique. J Arthroplasty 10: 313-8. http://doi.org/10.1016/s0883-5403(05)80180-9.

15. Muratli KS, Karatosun V, Uzun B, Celik S (2014) Subtrochanteric shortening in total hip arthroplasty: biomechanical comparison of four techniques. J Arthroplasty 29: 836-42. http://doi.org/10.1016/j.arth.2013.09.004.

16. Yan F, Chen G, Yang L, He R, Gu L, Wang F (2014) A reduction technique of arthroplasty without subtrochanteric femoral shortening osteotomy for the treatment of developmental high dislocation of hip: a case series of 28 hips. J Arthroplasty 29: 2289-93. http://doi.org/ 10.1016/j.arth.2013.11.016.

17. Wu X, Li SH, Lou LM, Cai ZD (2012) The techniques of soft tissue release and true socket reconstruction in total hip arthroplasty for patients with severe developmental dysplasia of the hip. Int Orthop 36: 1795-801. http://doi.org/10.1007/s00264-012-1622-6.

18. Li H, Yuan Y, Xu J, Chang Y, Dai K, Zhu Z (2018) Direct Leverage for Reducing the Femoral Head in Total Hip Arthroplasty Without Femoral Shortening Osteotomy for Crowe Type 3 to 4 Dysplasia of the Hip. J Arthroplasty 33: 794-99. http://doi.org/10.1016/j.arth.2017.09.011.

19. Lai KA, Liu J, Liu TK (1996) Use of iliofemoral distraction in reducing high congenital dislocation of the hip before total hip arthroplasty. J Arthroplasty 11: 588-93. http://doi.org/10.1016/s08835403(96)80114-8.

20. Imbuldeniya AM, Walter WL, Zicat BA, Walter WK (2014) Cementless total hip replacement without femoral osteotomy in patients with severe developmental dysplasia of the hip: minimum 15-year clinical and radiological results. Bone Joint J98-B: 1449-54. http://doi.org/10.1302/0301620X.96B11.33698.

21. Li H, Xu J, Qu X, Mao Y, Dai K, Zhu Z (2017) Comparison of Total Hip Arthroplasty With and Without Femoral Shortening Osteotomy for Unilateral Mild to Moderate High Hip Dislocation. J Arthroplasty 32: 849-56. http://doi.org/10.1016/j.arth.2016.08.021.

22. Nercessian OA, Macaulay W, Stinchfield FE (1994) Peripheral neuropathies following total hip arthroplasty.J Arthroplasty 9: 645-51. http://doi.org/10.1016/0883-5403(94)90119-8.

23. Higuchi $Y$, Hasegawa $Y$, Ishiguro $N(2015)$ Leg lengthening of more than $5 \mathrm{~cm}$ is a risk factor for sciatic nerve injury after total hip arthroplasty for adult hip dislocation. Nagoya J Med Sci 77: 45563.

24. Kerboull M, Hamadouche M, Kerboull L (2001) Total hip arthroplasty for Crowe type IV developmental hip dysplasia: a long-term follow-up study. J Arthroplasty 16: 170-6. http://doi.org/10.1054/arth.2001.28368.

25. Kong X, Chai W, Chen J, Yan C, Shi L, Wang Y (2019) Intraoperative monitoring of the femoral and sciatic nerves in total hip arthroplasty with high-riding developmental dysplasia. Bone Joint J101-B: 1438-46. http://doi.org/10.1302/0301-620X.101B11.BJJ-2019-0341.R2.

26. Xu H, Zhou Y, Liu Q, Tang Q, Yin J (2010) Femoral morphologic differences in subtypes of high developmental dislocation of the hip. Clin Orthop Relat Res 468: 3371-6. http://doi.org/ 
10.1007/s11999-010-1386-5.

27. Ma HY, Zhou YG, Zheng C,Cao WZ, Wang Sen, Wu WM, Piao S, Du YQ (2016) [New classification of Crowe type IV developmental dysplasia of the hip]. Zhongguo Gu Shang 29: 119-24.

28. Cooke TD, Scudamore RA, Bryant JT, Sorbie C, Siu D, Fisher B (1991) A quantitative approach to radiography of the lower limb. Principles and applications. J Bone Joint Surg [Br] 73: 715-20.

29. Zhou Y, Sun C, Wang Y (2012) New Method Addressing the Problem of Using Ceramic-on-Ceramic Bearing in Too Small Acetabulum of High-Riding DDH Patients with THA. Semin Arthro23: 226-31.

30. Ollivier M, Abdel MP, Krych AJ, Trousdale RT, Berry DJ (2016) Long-Term Results of Total Hip Arthroplasty With Shortening Subtrochanteric Osteotomy in Crowe IV Developmental Dysplasia. J Arthroplasty 31: 1756-60. http://doi.org/10.1016/j.arth.2016.01.049.

31. Wang D, Li LL, Wang HY, Pei FX, Zhou ZK (2017) Long-Term Results of Cementless Total Hip Arthroplasty With Subtrochanteric Shortening Osteotomy in Crowe Type IV Developmental Dysplasia. J Arthroplasty 32: 1211-19. http://doi.org/10.1016/j.arth.2016.11.005.

32. Zeng WN, Liu JL, Wang FY,Zhang X, Fan HQ, Chen GX, Guo L, Duan XJ, Zhou Q, Yang L (2017) Total hip arthroplasty for patients with Crowe type IV developmental dysplasia of the hip: Ten years results. Int J Surg 42: 17-21. http://doi.org/10.1016/j.ijsu.2017.04.029.

33. Argenson JN, Ryembault E, Flecher X, Brassart N, Parratte S, Aubaniac JM (2005) Three-dimensional anatomy of the hip in osteoarthritis after developmental dysplasia. J Bone Joint Surg [Br] 87: 1192-6. http://doi.org/10.1302/0301-620X.87B9.15928.

34. Brunner A, Ulmar B, Reichel H, Decking R (2008) The Eftekhar and Kerboul classifications in assessment of developmental dysplasia of the hip in adult patients. Measurement of inter- and intraobserver reliability. HSS J 4: 25-31. http://doi.org/10.1007/s11420-007-9066-z.

35. Li Y, Zhang X, Wang Q, Peng X, Wang Q, Jiang Y, Chen Y (2017) Equalisation of leg lengths in total hip arthroplasty for patients with Crowe type-IV developmental dysplasia of the hip: classification and management. Bone Joint J99-B: 872-79. http://doi.org/ 10.1302/0301-620X.99B7.BJJ-20161328.R1.

36. Nagoya S, Kaya M, Sasaki M, Tateda K, Kosukegawa I, Yamashita T (2009) Cementless total hip replacement with subtrochanteric femoral shortening for severe developmental dysplasia of the hip.J Bone Joint Surg [Br] 91: 1142-7. http://doi.org/10.1302/0301-620X.91B9.21736.

37. Fujishiro T, Nishiyama T, Hayashi S, Kurosaka M, Kanno T, Masuda T (2012) Leg length change in total hip arthroplasty with subtrochanteric femoral shortening osteotomy for Crowe type IV developmental hip dysplasia. J Arthroplasty 27: 1019-22. http://doi.org/ 10.1016/j.arth.2012.01.032.

\section{Figures}




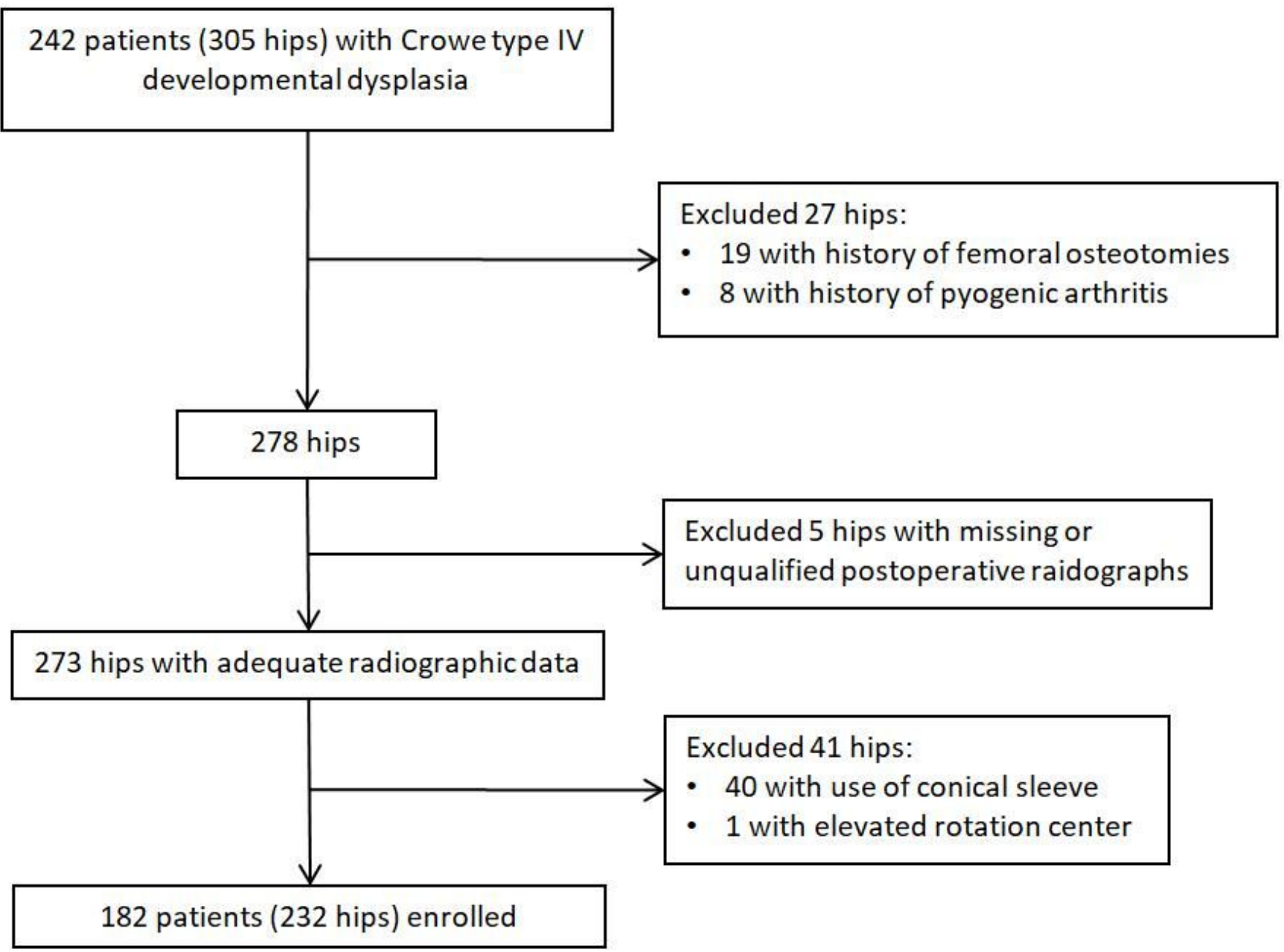

Figure 1

A flow diagram of the study
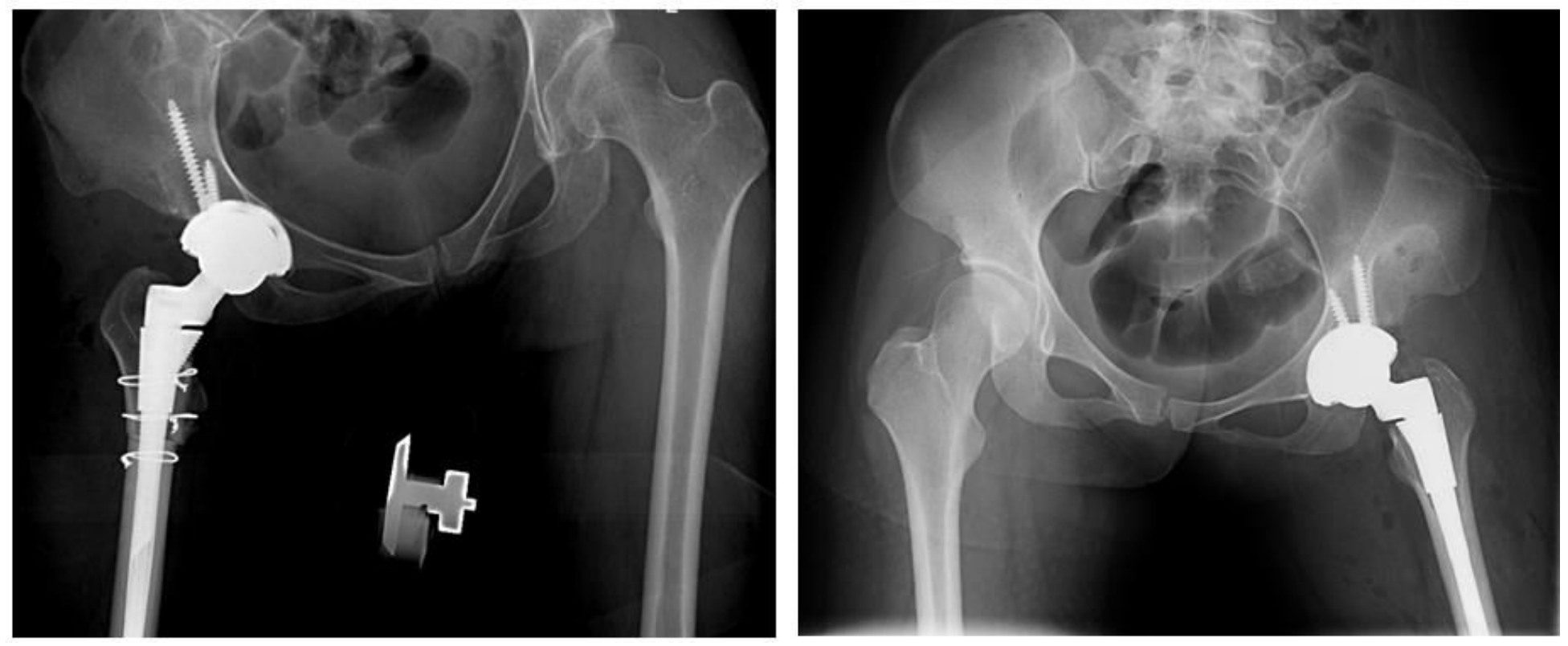

Figure 2 
The performance of subtrochanteric osteotomy was validated by osteotomy line on images of the second day after surgery. (Left) Use of subtrochanteric osteotomy. (Right) Without use of subtrochanteric osteotomy
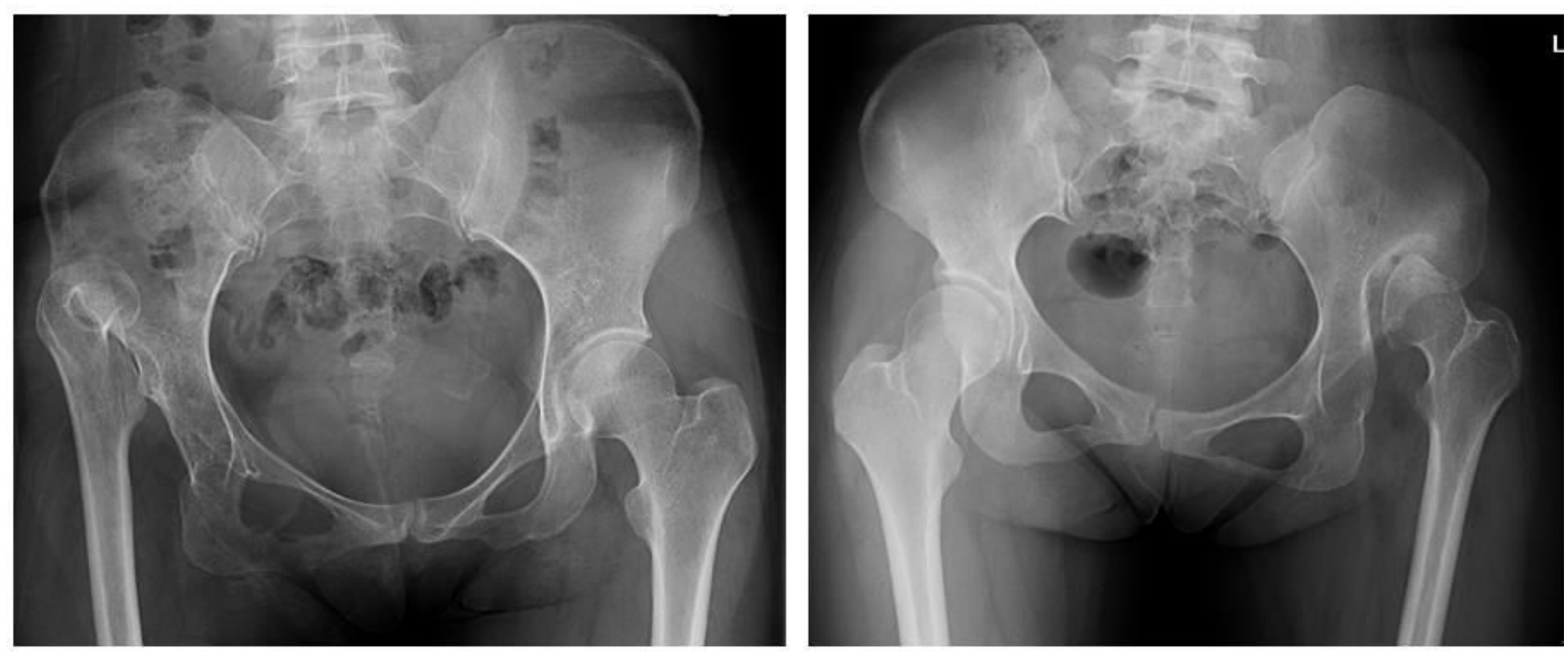

\section{Figure 3}

(Left) Absence of false acetabulum; (Right) false acetabulum formation 


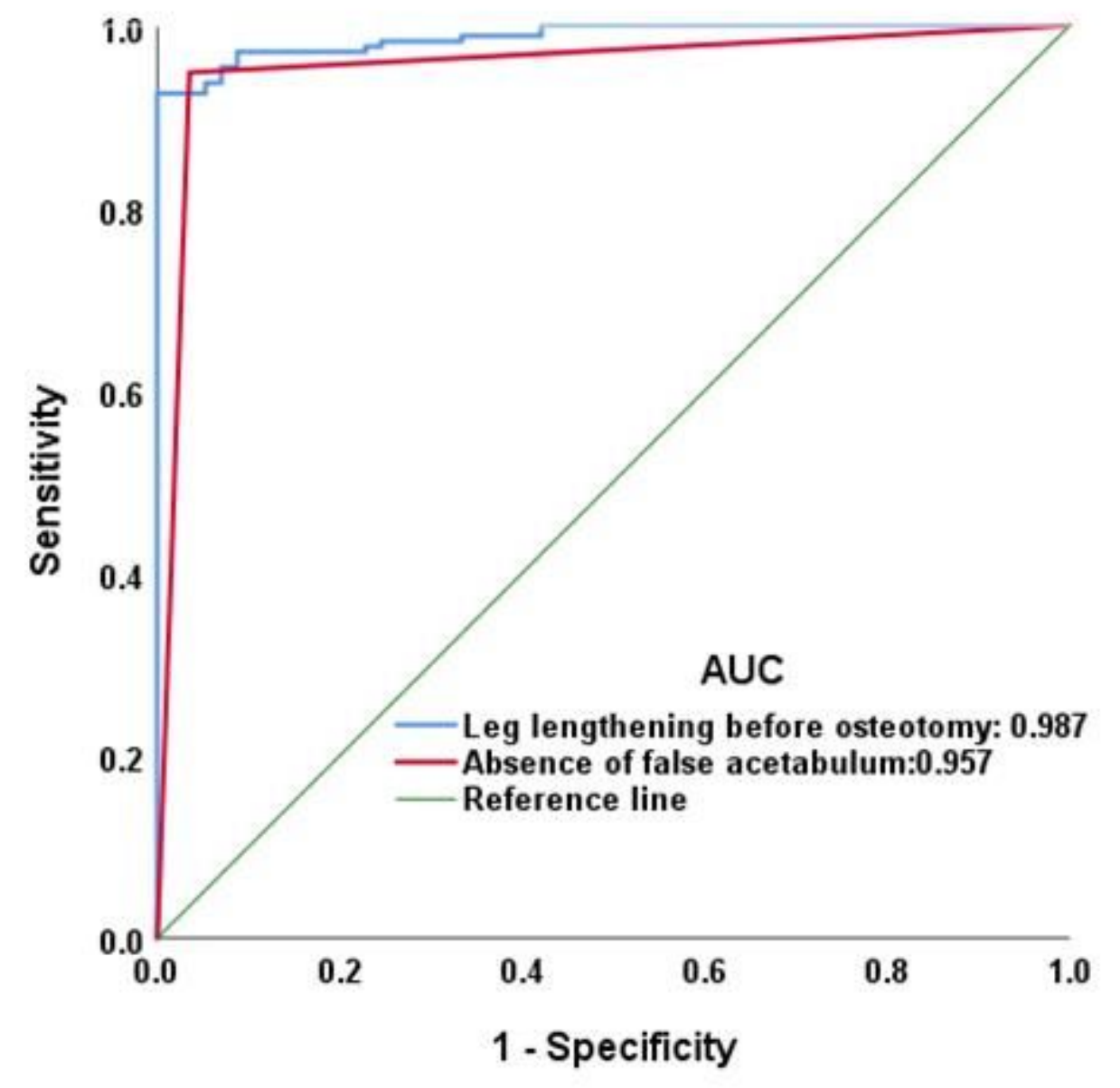

Figure 4

ROC curves for absence of false acetabulum and distalization of greater trochanter in predicting the use of subtrochanteric osteotomy

\section{Supplementary Files}

This is a list of supplementary files associated with this preprint. Click to download.

- 4.pdf

- 3.pdf

- 5.pdf

- 1.pdf

- 6.pdf

- 2.pdf 